\title{
Analysis of Calibration Data for the Uranium Active Neutron Coincidence Counting Collar with Attention to Errors in the Measured Neutron Coincidence Rate
}

\author{
Stephen Croft ${ }^{1}$, Tom Burr ${ }^{2}$, Andrea Favalli ${ }^{3}$, and Andrew Nicholson ${ }^{1}$ \\ ${ }^{1}$ Oak Ridge National Laboratory (ORNL), One Bethel Valley Road, Oak Ridge, TN, US \\ ${ }^{2}$ International Atomic Energy Agency (IAEA), Vienna (Austria) \\ ${ }^{3}$ Los Alamos National Laboratory (LANL), MS E540, Los Alamos, NM, 87545, US
}

\begin{abstract}
The declared linear density of ${ }^{238} \mathrm{U}$ and ${ }^{235} \mathrm{U}$ in fresh low enriched uranium light water reactor fuel assemblies can be verified for nuclear safeguards purposes using a neutron coincidence counter collar in passive and active mode, respectively. The active mode calibration of the Uranium Neutron Collar - Light water reactor fuel (UNCL) instrument is normally performed using a non-linear fitting technique. The fitting technique relates the measured neutron coincidence rate (the predictor) to the linear density of ${ }^{235} \mathrm{U}$ (the response) in order to estimate model parameters of the nonlinear Padé equation, which traditionally is used to model the calibration data. Alternatively, following a simple data transformation, the fitting can also be performed using standard linear fitting methods. This paper compares performance of the nonlinear technique to the linear technique, using a range of possible error variance magnitudes in the measured neutron coincidence rate. We develop the required formalism and then apply the traditional (nonlinear) and alternative approaches (linear) to the same experimental and corresponding simulated representative datasets. We find that, in this context, because of the magnitude of the errors in the predictor, it is preferable not to transform to a linear model, and it is preferable not to adjust for the errors in the predictor when inferring the model parameters.
\end{abstract}

Key words: Neutron Collar; Fresh LWR Fuel Assay; Linear Regression; Errors in Predictor 


\section{Introduction and Background}

A type of neutron coincidence collar in common use is typically referred to as UNCL, the Uranium Neutron Collar - light water reactor (LWR) fuel, an acronym which succinctly describes both the instrument and its principle function in international nuclear safeguards. Collars of this family are routinely used by nuclear safeguards inspectors to verify the uranium content in fresh (unirradiated) LWR fuel assemblies [1-8]. In passive neutron coincidence counting mode, the linear density $\left(\mathrm{g}{ }^{238} \mathrm{U} \cdot \mathrm{cm}^{-1}\right)$ of ${ }^{238} \mathrm{U}$ can be inferred through its spontaneous fission coincidence neutron signature. In the active mode, an AmLi radionuclide source is used to induce slow-neutron fission and the ${ }^{235} \mathrm{U}$ linear density $\left(\mathrm{g}{ }^{235} \mathrm{U} \cdot \mathrm{cm}^{-1}\right)$ in the fuel assembly is inferred from an analysis of the net reals coincidence rate (that is, the genuine neutron coincidence corrected for accidental or chance pile-up coincidences, and also corrected for background coincidences coming from the fuel and the environment) observed using shiftregister time correlation counting logic [9-10].

It is traditional practice $[3,4]$ to represent the active response of UNCL non-destructive assay instruments empirically using a Padé approximation [11-12]. Denoting the reals coincidence rate (net doubles corrected for both chance coincidences and passive background) by $R$, and the linear mass density of ${ }^{235} \mathrm{U}$ by $m$, the traditional form used to describe the calibration data is:

$$
R=\frac{a m}{1+b m}
$$

with calibration model parameters $a>0$ and $b>0$ for the problem at hand When $b \cdot m \ll 1$ equation (1) simplifies to a proportionate response, while in the other limit, $b \cdot m \gg 1$, it predicts that $R \rightarrow a / b$.

As commonly practiced the calibration data is represented according to equation (1), that is $R$ is described as a function of $m$, and non-linear fitting without uncertainty in the $m$ values is applied to estimate the model parameters. However, the main goal of the measurement is to estimate $m$, not $R$. So, upon re-arranging, and after introducing generic notation $x$ for the predictor $(R)$ and $y$ for the response $(\mathrm{m})$, we elect to work with the following measurement expression:

$$
y(x)=\frac{a_{1} x}{1+a_{2} x}
$$

where $y$ is the linear density of ${ }^{235} \mathrm{U}$ in $\mathrm{g}{ }^{235} \mathrm{U} \cdot \mathrm{cm}^{-1}, x$ is the background corrected reals coincidence rate in units of doubles $\cdot \mathrm{s}^{-1}$, and $a_{1}\left(\mathrm{~g}^{235} \mathrm{U} \cdot \mathrm{cm}^{-1} \cdot\right.$ doubles $\left.^{-1} \cdot \mathrm{s}\right)$ and $a_{2}\left(\right.$ doubles $\left.^{-1} \cdot \mathrm{s}\right)$ are model parameters determined from calibration data. In terms of the original framing of the problem in terms of the parameters $a$ and $b$, we have $a_{1}=1 / a$ and $a_{2}=-b / a<0$. The underlying physics of the problem requires that $y, x$, and $a_{1}$ are all positive, while $a_{2}$ is negative over the range of practical application. This serves as a constraint in our analysis (but we did not have to enforce this constraint because it was satisfied in all real and simulated data). Because we are not using a Bayesian approach, we regard the true linear density of ${ }^{235} \mathrm{U}$ as an unknown constant, denoted as $y$. Regarding notation, we use $X$ to denote the measured neutron 
coincidence rate, which is a random variable. In equation (2), we regard $x$ as a true (mean) value of the random variable $X$. We will be fitting $y$ to $X$, so errors in $X$ must also be considered.

The function $y(x)$ has a simple smooth shape that is monotonically increasing from the origin to an asymptotic value. When $\left|a_{2} x\right| \ll 1, y \approx a_{1} x$, a proportionate response with slope $a_{1}$. In the limit $a_{2} x \rightarrow-1, y \rightarrow \infty$. Intuitively, and also quantitatively based on detailed neutron transport simulations, we do not expect the response to follow this shape exactly over a wide dynamic range, and in particular we would not expect the doubles response to plateau with linear density (i.e. $R \rightarrow a / b=-1 / a_{2}$ in the limit $m \rightarrow \infty$ ). This is because even when all the available interrogating neutrons are absorbed the doubles will continue to grow due to induced fission multiplication. In the first published works on UNCL application a quadratic function between R and $\mathrm{m}$ [1] was used, and a power function with exponent positive and less than unity [2] has been applied. But since reference [3], the Padé approximation has been adopted and used exclusively as the convenient, but otherwise arbitrary, functional form to represent the response over the limited dynamic range of practical interest characteristic of commercial power reactors. Usually the uranium in Light Water Reactor (LWR) fuel is lightly enriched above the natural isotopic ratio and below a licensing limit of $5 \mathrm{wt} \%$. Assembly design varies between reactor types (pin diameter, cladding, number of pins, spacing etc.) but from a neutronics perspective all are broadly similar within a reactor class (e.g. pressurized water reactor or boiling water reactor).

This paper also develops an approach for estimating the model parameters that uses the more familiar linear fitting methods rather than non-linear methods. We develop the required formalism and then apply the traditional (nonlinear) and alternative approaches (linear) to the same actual experimental and corresponding simulated representative datasets. We also consider the impact of measurement error in $X$ (the doubles count rate). Note that any model bias due to the assumed Padé shape that is present in the real data increases the estimated error variance; and, this increased error variance is used in the corresponding simulated data. Because the Padé shape has no physical basis, model mismatch (never formally evaluated previously to the best of our knowledge), is a potential source of bias in the assay results. We end by discussing how the calibration is to be applied to actual field measurements, and how error in the predictor parameters is best propagated into the final assay results of practical interest.

\section{Methods}

First we list the partial derivatives from equation (2) because they will be needed later:

$$
\begin{aligned}
& \frac{1}{y} \frac{\partial y}{\partial x}=\frac{y}{a_{1} x} \frac{1}{x} \\
& \frac{1}{y} \frac{\partial y}{\partial a_{1}}=\frac{1}{a_{1}} \\
& \frac{1}{y} \frac{\partial y}{\partial a_{2}}=\frac{-y}{a_{1} / a_{2}} \frac{1}{a_{2}}
\end{aligned}
$$

The form of $y(x)$ is non-linear in the parameters but by algebraic rearrangement we obtain a linear relationship as follows: 


$$
z(t)=\frac{1}{y}=\frac{1+a_{2} x}{a_{1} x}=\frac{a_{2}}{a_{1}}+\frac{1}{a_{1}} \frac{1}{x}=\beta_{1}+\beta_{2} t
$$

where $z=1 / y, t=1 / x, \beta_{1}=a_{2} / a_{1}$, and $\beta_{2}=1 / a_{1}$.

The parameters $\beta_{1}$ and $\beta_{2}$ may now be estimated from the measured calibration data set using familiar weighted linear least squares (WLS) [13-16] fitting techniques that accommodate uncertainties in both coordinates (that is in both the fuel assembly loading specifications and in the observed counting rates). This is an important point because the horizontal axis (the measured neutron coincidence rate) is subject to experimental uncertainty arising from nuclear counting statistics, detector positioning, background determination, wrapper corrections and other practical factors. An unweighted (ordinary least squares, OLS) linear fit would be suboptimal because it would not correctly represent the contribution of each point in the untransformed space [17]. The WLS fit with uncertainties in both coordinates is analytical and is straightforward and convenient to implement in, for example, a spreadsheet application [13-15]. It is exact from the perspective of minimizing the normalized distance of the points to the fitted line, and in the setting of the present discussion, is statistically equivalent to a non-linear fit provided the first order Taylor expansion used to transform the uncertainty structure in the data set is valid. Unlike non-linear methods, the linear solution does not require an initial estimate to be made for the parameters being determined. Certainly if the linear approach performs well compared to the nonlinear approach, it is to be preferred on the grounds of simplicity, a consideration particularly in the training context.

The calibration data $(x, y)$ is transformed as follows:

$$
\begin{aligned}
& t=\frac{1}{x} \\
& \left(\frac{\sigma_{t}}{t}\right)^{2}=\left(\frac{\sigma_{x}}{x}\right)^{2} \\
& z=\frac{1}{y} \\
& \left(\frac{\sigma_{z}}{z}\right)^{2}=\left(\frac{\sigma_{y}}{y}\right)^{2}
\end{aligned}
$$

Applying the usual rules of propagation of variance (POV) using first order Taylor series expansion [18-20] we have:

$$
\begin{aligned}
& a_{1}=\frac{1}{\beta_{2}} \\
& a_{2}=\frac{\beta_{1}}{\beta_{2}}
\end{aligned}
$$




$$
\begin{gathered}
\left(\frac{\sigma_{\hat{a}_{1}}}{a_{1}}\right)^{2}=\left(\frac{\sigma_{\hat{\beta}_{2}}}{\beta_{2}}\right)^{2} \\
\left(\frac{\sigma_{\hat{a}_{2}}}{a_{2}}\right)^{2}=\left(\frac{\sigma_{\hat{\beta}_{1}}}{\beta_{1}}\right)^{2}+\left(\frac{\sigma_{\hat{\beta}_{2}}}{\beta_{2}}\right)^{2}-2 \frac{\operatorname{cov}\left(\hat{\beta}_{1}, \hat{\beta}_{2}\right)}{\beta_{1} \beta_{2}} \\
\frac{\operatorname{cov}\left(\hat{a}_{1}, \hat{a}_{2}\right)}{a_{1} a_{2}}=\left(\frac{\sigma_{\hat{\beta}_{2}}}{\beta_{2}}\right)^{2}-\frac{\operatorname{cov}\left(\hat{\beta}_{1}, \hat{\beta}_{2}\right)}{\beta_{1} \beta_{2}}
\end{gathered}
$$

Note that the estimated model parameters are themselves random quantities, subject to small changes in future calibrations. Therefore, we follow convention and write for example, $\hat{a}_{1}$ to denote the estimate of $a_{1}$. Having established the calibration parameters we may now use them to perform an assay of a fuel assembly. Thus:

$$
\hat{y}(x)=\frac{\hat{a}_{1} x}{1+\hat{a}_{2} x}
$$

at the $x$ values used in calibration. For future measured $X$ values, we also use:

$$
\hat{y}(X)=\frac{\hat{a}_{1} X}{1+\hat{a}_{2} X}
$$

and we have a choice to use WLS estimates for $\hat{a}_{1}$ and $\hat{a}_{2}$, or to use modified WLS estimates that account for errors in both $X$ and $y$ in the calibration data [13]. Recall, if there is negligible error in $y$, there still could be inherent model error arising from use of the Padé shape, which in this context has the same qualitative effect as error in $y$. If there is structure (such as trends in the estimated errors in $Y$ among the $X$ values) in the model error, then alternate shapes should be considered.

Then, by POV, one would write for the fractional variance in the assay value:

$$
\left(\frac{\sigma_{y}}{y}\right)^{2}=\left(\frac{1}{y} \frac{\partial y}{\partial x} \sigma_{x}\right)^{2}+\left(\frac{1}{y} \frac{\partial y}{\partial a_{1}} \sigma_{\hat{a}_{1}}\right)^{2}+\left(\frac{1}{y} \frac{\partial y}{\partial a_{2}} \frac{\sigma_{\hat{a}_{2}}}{a_{2}}\right)^{2}+2 \frac{1}{y} \frac{\partial y}{\partial a_{1}} \frac{1}{y} \frac{\partial y}{\partial a_{2}} \operatorname{cov}\left(\hat{a}_{1}, \hat{a}_{2}\right)
$$

Substituting our earlier result and working in terms of the $a$-parameters this reduces to:

$$
\left(\frac{\sigma_{y}}{y}\right)^{2}=\left(\frac{y}{a_{1} x} \frac{\sigma_{x}}{x}\right)^{2}+\left(\frac{\sigma_{\hat{a}_{1}}}{a_{1}}\right)^{2}+\left(\frac{y}{a_{1} / a_{2}} \frac{\sigma_{\hat{a}_{2}}}{a_{2}}\right)^{2}-2 \frac{y}{a_{1} / a_{2}} \frac{\operatorname{cov}\left(\hat{a}_{1}, \hat{a}_{2}\right)}{a_{1} a_{2}}
$$


Evaluation of $\left(\frac{\sigma_{\hat{a}_{1}}}{a_{1}}\right)^{2},\left(\frac{\sigma_{\hat{a}_{2}}}{a_{2}}\right)^{2}$, and $\frac{\operatorname{cov}\left(\hat{a}_{1}, \hat{a}_{2}\right)}{a_{1} a_{2}}$ in terms of the $\beta$-parameters is carried out according to Eqns. 13-15.

The contributions coming from the uncertainty in the model parameters may be considered systematic at the particular measurement position and the covariance term arises because the two model parameters are estimated from the same set of calibration data. Reference [15] explains that in safeguards applications, it is necessary to distinguish random from systematic errors because safeguards error propagation applications are usually applied across multiple measured items. The Guide to the Expression of Uncertainty in Measurement [21] endorses the use of systematic ("correlated") errors in its variance propagation, although random and systematic errors variances are then combined to estimate a total uncertainty for an individual measurand.

\section{Example}

For illustration, we use a data set adapted from the historical No-Cd (thermal) mode calibration of the reference pressurized water reactor (PWR) neutron collar (LANL-3 with AmLi source MRC-95 installed) of revised (UNCL-II) design. The measurements were conducted at Los Alamos National Laboratory for the purpose of establishing the shape of the response as a function of enrichment to be adopted for all UNCLs [4].

The known calibration assemblies consisted of a $15 \times 15$ array of which 21 were guide tube locations leaving (15x15-21) fuel pin locations. Two types of uranium dioxide fuel pin were used. In the low enriched Uranium (LEU) set the ${ }^{235} \mathrm{U}$ to ${ }^{\text {tot }} \mathrm{U}$ enrichment was $3.194 \mathrm{wt} \%$ and in the depleted Uranium (DU) set it was $0.219 \mathrm{wt} \%$. To vary ${ }^{235} \mathrm{U}$ linear density LEU pins were replaced by DU pins uniformly across the fuel assembly. In this way commercial reactor fuel of about $3.2 \mathrm{wt} \%$ enrichment and below could be simulated. For this data set the total heavy metal (HM) linear density therefore remains at approximately $1220 \mathrm{~g} \cdot \mathrm{cm}^{-1}$ for all of the measured configurations.

The $9 y$ values (linear density of $\left.{ }^{235} \mathrm{U}\right)$ are $(16.19799,21.89250,27.58702,29.36656,31.14610$, $33.28154,34.70517,36.84061,38.97606)$. The 9 corresponding $x$ values (background corrected reals coincidence rate) are $(111.1,132.0,149.7,158.8,164.1,173.4,176.0,180.8,186.5)$, with absolute standard deviations in the measured $X$ values of $0.74,0.74,0.76,0.79,0.80,0.83,0.83$, 0.84 , and 0.35 , respectively. Note the linear density values listed, although based on the work of [4] are not exactly those reported in [4] because we have used our own assessment of the fuel specification. For the present purposes this is of no consequence.

Figure 1a plots y versus $x$ and shows the least squares linear fit of $y$ to $x$ and also the fitted nonlinear Padé approximation. Figure $1 \mathrm{~b}$ shows the relative errors $\frac{y-\hat{y}}{y}$ for the linear and nonlinear fits. For this calibration data, the root mean squared error defined in the usual way as 
$R M S E=\sqrt{\sum_{i=1}^{9}\left(y_{i}-\hat{y}_{i}\right)^{2} / 9}$ in estimating $y$ is 0.61 for the linear fit. It is 0.53 for the nonlinear fit.

Therefore, because 0.61 is not dramatically larger than 0.53 , and because there are no clear patterns in the residuals from the linear fit evident in Figure 1b, one could consider simply fitting $y$ to $x$ rather than $1 / y$ to $1 / x$ for this particular calibration data set,.

\section{\{HERE FIG. 1\}}

\subsection{Simulation Study}

To assess performance in estimating $y$, we performed a simulation study using the fitted Padé model as the true model. One reason to do a simulation study is that it provides a simple and defensible basis to assess estimation performance. From this (assumed) true model we simulate hypothetical $(Y, X)$ pairs to be used in hypothetical calibrations. Each $X$ value is simulated from a Gaussian distribution (an arbitrary but reasonable assumption here), with mean $x$ (using each of the 9 real $x$ values in the original calibration data) and standard deviation $\sigma_{\mathrm{X}}$. Each $\mathrm{Y}$ value is simulated from a Gaussian with mean $\frac{\hat{a}_{1} x}{1+\hat{a}_{2} x}$ and standard deviation 0.53 . Recall that the 0.53 value is the standard deviation in the 9 residuals in the nonlinear fit to the training data; because this includes the impact of errors in $X$, it is a slight overestimate, but is adequate for our simulation study for the purpose of comparing data treatment options. This will of course favor using the fitted Padé model over any alternative, because the fitted Padé model is the (assumed) true model. If we were doing simple WLS without error in X, we could rely on analytical results to estimate the RMSE arising from applying the estimate $\frac{\hat{a}_{1} x}{1+\hat{a}_{2} x}$, where $\hat{a}_{1}=0.11$ and $\hat{a}_{2}=-0.0253$ are estimates in future calibrations using the same 9 true $(y, x)$ pairs.

We considered both the nonlinear Padé fitting applied to $y(x)=\frac{a_{1} x}{1+a_{2} x}$ and also the linear fitting described previously, using $z(t)=\frac{1}{y}=\frac{1+a_{2} x}{a_{1} x}=\frac{a_{2}}{a_{1}}+\frac{1}{a_{1}} \frac{1}{x}=\beta_{1}+\beta_{2} t$. We used both LS that ignored errors in $\mathrm{X}$, seeking to minimize $R S S_{1}={ }_{i=1}^{9}\left(y_{i} \frac{a_{1} x}{1+a_{2} x}\right)^{2} / 0.53^{2}$ and also used a WLS procedure that considered errors in $\mathrm{X}$ and $Y$, seeking to minimize $R S S_{2}=\sum_{i=1}^{9}\left(y_{i}-\frac{a_{1} x}{1+a_{2} x}\right)^{2} / 0.53^{2}+\left(x_{i}-\hat{x}_{i}\right)^{2} / \sigma_{x i}^{2}$. We used the nlminb function in $\mathrm{R}$ [22] to find the $\hat{a}_{1}$ and $\hat{a}_{2}$ that minimized $R S S_{1}$ or $R S S_{2}$. Because we did not have an estimate of $\sigma_{y i}^{2}$ separately for each of the $9 y_{\mathrm{i}}$ values, the LS procedure assumes equal variance, of $0.2809=$ $0.53^{2}$. For each of $10^{5}$ simulated sets of the $9(Y, X)$ calibration pairs, we assumed we had separate testing data, arbitrarily chosen to be at the same $9 X$ values as in calibration (so there is no extrapolation in going from training/calibration to testing). The number of simulations is so large $\left(10^{5}\right)$, that random error due to having a finite number of simulations is negligible. 
Figure 2 shows a plot the estimated random error standard deviation at each of the 9 testing locations. In Figure $2 \mathrm{a}$, the WLS option that adjusts for error in the predictor is used $\left(\mathrm{RSS}_{2}\right)$. In Figure $2 b$, the LS procedure that does not adjust for error in the predictor is used $\left(\mathrm{RSS}_{1}\right)$. Figure $2 \mathrm{c}$ is the same as Figure $2 \mathrm{a}$, but is for the systematic error standard deviation. Figure $2 \mathrm{~d}$ is the same as Figure $2 b$, but is for the systematic error standard deviation. The random and systematic error standard deviations are estimated by fitting $\hat{a}_{1}$ and $\hat{a}_{2}$ in each of $10^{5}$ simulations. For completeness, the set of $10^{5}$ simulations was repeated several times to confirm that $10^{5}$ was sufficiently large to ignore variation due to having a finite number of simulations. Figure 2 plots estimates of the random and systematic error standard deviations from each of two such sets of $10^{5}$ simulations. A numerical summary is provided in the next subsection.

\subsection{Simulation Study Results}

The root mean squared estimation error (RMSE) is 0.36 whether we adjust for errors in the predictor $\left(\mathrm{RSS}_{2}\right)$ or not $\left(\mathrm{RSS}_{1}\right)$. The reason that the RMSE is the same in fitting the Padé model, whether we adjust for errors in the predictor or not, is simply that the predictor error standard deviation is small. To illustrate this, if we multiply the predictor error standard deviation by a factor of 10 simply, the RMSE becomes 2.67 if we adjust for errors in the predictor, and 2.45 if we do not adjust for errors in the predictor. All RMSE results are repeatable to within \pm 0.01 . There is no evidence of overall long-term bias (-0.01 with a standard deviation of 0.006 or -0.1 with a standard deviation of 0.06 ).

If we transform to a linear model as described, and again if we multiply the predictor error standard deviation by a factor of 10 simply for illustration, then the RMSE increases to more than 1.0 if we adjust for errors in the predictor, and to 0.42 if we do not adjust for errors in the predictor. One reason for the poor performance of the transform-to-linear option is that the errors in the predictor are amplified when the predictor is $1 / x$ rather than $x$. Another reason is that we seek to estimate $y$, not $1 / y$, so we have to transform back to predict $y$, which does introduce long-term bias. For example: with the nominal values of the absolute standard deviations in the measured $X$ values give above, we observe a bias of 0.04 (with a standard deviation of 0.01 across simulations) for the sum of the 9 test item responses in the case of not adjusting for errors in the predictor, and a bias of 82 (with a standard deviation of 25 across simulations) for the sum of the 9 test items in the case of adjusting for the errors in the predictor.

\section{\{HERE FIG.2\}}

This study shows that it is preferable to work with the original $(x, y)$ pairs and fit the Padé model, rather than transform to $1 / y$ and $1 / x$. However, for completeness here, we also assessed the quality of the approximations given in Section 2 for $\left(\frac{\sigma_{\hat{a}_{1}}}{a_{1}}\right)^{2},\left(\frac{\sigma_{\hat{a}_{2}}}{a_{2}}\right)^{2}$, and $\frac{\operatorname{cov}\left(\hat{a}_{1}, \hat{a}_{2}\right)}{a_{1} a_{2}}$. In the $10^{5}$ 
simulations, the average estimated value of $\left(\frac{\sigma_{\hat{a}_{1}}}{a_{1}}\right)^{2},\left(\frac{\sigma_{\hat{a}_{2}}}{a_{2}}\right)^{2}$, and $\frac{\operatorname{cov}\left(\hat{a}_{1}, \hat{a}_{2}\right)}{a_{1} a_{2}}$ are $0.0005,0.001$, and -0.0008 , respectively. The corresponding observed values were $0.001,0.002$, and -0.0017 , respectively (repeatable across sets of $10^{5}$ simulations to the number of digits shown). This shows reasonable but not perfect agreement for the case with the nominal (almost negligible) error standard deviations in $X$.

\section{Discussion and Conclusion}

Recall that this numerical study assumed the Padé model with the WLS fitted parameters was the true model; in that case, it is better not to transform to a model that is linear in the parameters. And, this UNCL calibration is an example where a method to adjust for errors in the predictor is not helpful (and can in fact be harmful), because the goal is to predict future test $y$ values using future $X$ values. The method that does not adjust for errors in the predictor leads a consistent estimator of the parameter required for best linear predictor of $y$ given $x$. To clarify, assume that $(X, Y)$ have a bivariate normal distribution, that $X=x+R_{X}, Y=y+R_{Y}=\beta_{0}+\beta_{1} x+R_{Y}$, then the

best linear predictor of $\mathrm{Y}$ given $\mathrm{X}$ is $\mu_{Y}+\rho \frac{\sigma_{Y}}{\sigma_{X}}\left(X-\mu_{X}\right)$, where $\rho$ is the correlation between $X$ and $Y$. And, the least squares estimate in the regression of $\mathrm{Y}$ on $\mathrm{X}$ converges for large samples to $\frac{\operatorname{cov}(X, Y)}{\sigma_{X}^{2}}=\frac{\beta_{1} \sigma_{x}^{2}}{\sigma_{x}^{2}+\sigma_{R x}^{2}}$, which is the desired value because $\rho \frac{\sigma_{Y}}{\sigma_{X}}=\frac{\operatorname{cov}(X, Y)}{\sigma_{X}^{2}}$. Note that the value $\frac{\beta_{1} \sigma_{x}^{2}}{\sigma_{x}^{2}+\sigma_{R x}^{2}}$ is an attenuation toward zero of the true $\beta_{1}$ value. Therefore, a method that adjusts for errors in the predictor $X$ is helpful (and one example of such a method is Deming regression, which is implemented in neutron coincidence counting software [4]) only if interest is on the true $\beta_{1}$ value rather than on predicting future test $y$ values. The same reasoning applies in the case where the true model is nonlinear such as the Padé model; we conclude that one should not adjust for errors in the predictor. Recall that in the simulations that used errors with very small magnitudes in the predictor (corresponding to those in the example data), there was no meaningful difference in performance if we adjusted for EIP or not. In the simulations with increased error magnitudes in the predictor (which can arise, for example, when the predictor is adjusted to adjust test items to calibration items as in [6]), it was clearly preferable not to adjust for EIP.

One related question is whether the measurement error in the $X$ 's will be the same in test data as in training data. In most practical situations, the measurement error variance in the X's in test data will be the same as or larger than in training data. If it is the same, then the simulation strategy in Section 3 is appropriate. If it is larger, then one can artificially increase the error variance in the X's in the training data in order to match that in the testing data, and re-estimate the regression parameters. 


\section{Acknowledgement}

\section{The work described in this paper was funded by the U.S. DOE under action sheet AS-16 between The National Nuclear Energy Commission of Brazil (CNEN) and the United States Department of Energy (DOE).}

\section{References}

[1] H.O. Menlove, Description and performance characteristics for the neutron coincidence collar for the verification of reactor fuel assemblies, Los Alamos National Laboratory report LA-8939-MS (Aug., 1981). Also ISPO-142.

[2] H.O. Menlove, M.A.S. Marzo, SG.de Almeida, M.C.de Almeida, L.P.M. Moitta, L.F. Conti , and J.R.T. de Paiva, In-Plant Test and Evaluation of the Neutron Collar for Verification of PWR Fuel Assemblies at Resende, Brazil, LA-10562-MS (Nov.1985).

[3] H.O. Menlove and J.E. Pieper, Neutron collar calibration for assay of LWR fuel assemblies, Los Alamos National Laboratory Report LA10827-MS. Also known as ISPO-258 (March, 1987).

[4] H.O. Menlove, JE Stewart, S.Z. Qiao, T.R. Wenz, and G.P.D. Verrecchia, Neutron Collar Calibration and Evaluation for Assay of LWR Fuel Assemblies Containing Burnable Neutron Absorbers, Los Alamos National Laboratory report LA-11965-MS (1990). Issued Nov. 1990. Also known as ISPO-323.

[5] S. Croft, A. Favalli, M.T. Swinhoe, C. D Rael, State of the Art Monte Carlo modeling of active collar measurements and comparison with experiment, Proc. 52nd Annual INMM Meeting, July 2011 Palm Desert, CA, USA.

[6] A. Favalli, S. Croft, M.T. Swinhoe, Perturbation and burnable poison rod corrections for BWR uranium neutron collar, Proc. 33rd ESARDA Annual meeting, Helia Conference Hotel, Budapest, Hungary, 16-20 May, 2011.

[7] S. Croft, A. Favalli, and M.T. Swinhoe, The calculation of burnable poison corrections factors for PWR fresh fuel active collar measurements, $53^{\text {rd }}$ INMM Annual Meeting 2012, Orlando, USA.

[8] M. Looman, R. Jaime, P. Peerani, P. Shchillebeeckx, S. Jung, P. Schwalbach, M.T. Swinhoe, P.J. Chare and W.F. Kloeckner, The Effect of Gadolinium Poison Rods on the Active Neutron Measurement of LEU Fuel Assemblies, ESARDA 2001, Bruges (Belgium).

[9] D. Reilly, N. Ensslin, H. Smith, Jr., and S. Kreiner, Passive nondestructive assay of nuclear materials, United States Nuclear Regulatory Commission Report NUREG/CR-5550, 1991

[10] S. Croft, A. Favalli, D. Hauck, D. Henzlova, and P. Santi, Feynman Variance-to-mean in the context of passive neutron coincidence counting, Nuclear Instruments and Methods, Vol.686, 2012, pp. 136-144.

[11] C.F. Gerald, Applied Numerical Analysis, Addison-Wesley Publishing Company (second printing, 1980). ISBN 0-201-02969-1.

[12] C.F. Gerald and P.O. Wheatley, Applied Numerical Analysis (7th edition), Addison-Wesley (2003). ISBN-13: 978-0321133045.

[13] T. Burr, S. Croft, and B.C Reed, Least-Squares Fitting with Errors in the Response and Predictor, International Journal of Metrology and Quality Engineering 3(2012)117-123.

[14] S. Croft, T. Burr, and A. Favalli, A simple-minded direct approach to estimating the calibration parameter for proportionate data, Radiation Measurements 47(2012)486-491.

[15] S. Croft and T. Burr, Calibration of Nondestructive Assay Instruments: An Application of Linear Regression and Propagation of Variance, Applied Mathematics (AM)5(2014)785-798, Special Issue on "Regression Model”, Guest Editor Prof. Chris Cannings, University of Sheffield,

UK.http://dx.doi.org/10.4236/am.2014.55075 (http://www.scirp.org/iournal/am), (AM, ISSN: 2152-7393), a peer-reviewed open access journal.

[16] S. Croft, T.L. Burr, and A. Favalli, Estimating the half-life of ${ }^{241} \mathrm{Pu}$ and its uncertainty, Radiation Measurements 59(2013)94-102.

[17] S. Chatterjee and J.S. Simonoff, Handbook of regression analysis, John Wiley \& Sons (2013). ISBN 978-0-470-88716-5

[18] J.M. Kirkpatrick, S. Croft, T. Burr, M. Soriano, and R. Venkataraman, Fundamental considerations in uncertainty analysis for NDA measurements, Proceeding of the 55th Annual Meeting of the Institute of Nuclear Materials Management, July 20-24, 2014, Atlanta, Georgia, USA

[19] S. Croft, T. Burr, K.D. Jarman, J.M. Kirkpatrick, and R Venkataraman, Exact propagation of variance and confidence interval calculations, Proceeding of the 55th Annual Meeting of the Institute of Nuclear Materials Management, July 20-24, 2014, Atlanta, Georgia, USA. 
[20] S. Croft, and A. Favalli, How the choice of data reduction can strongly influence the uncertainty assessment: A re-analysis of Mn-bath experiments; Radiation Measurements (2012), Vol.47, Issue 7, 2012, pp.481-485.

[21] Evaluation of Measurement Data - Guide to the Expression of Uncertainty In Measurement, Joint Committee for Guides in Metrology report JCGM 100, (First Edition, 2008) Corrected Version (2010), www.bipm.org.

[22] R DEVELOPMENT CORE TEAM, R: A Language and Environment for Statistical Computing, R Foundation for Statistical Computing: http://www.R-project.org (2013).

\section{Figure Captions}

Figure 1. The $9(y, x)$ pairs and the corresponding linear and nonlinear fits.

Figure 2. The estimated random and systematic error standard deviation at each of the 9 test values of $x$. In (a) and (c), there is an adjustment for errors in the predictor (EIP). In (b) and (d), there is no adjustment for errors in the predictor (EIP). 


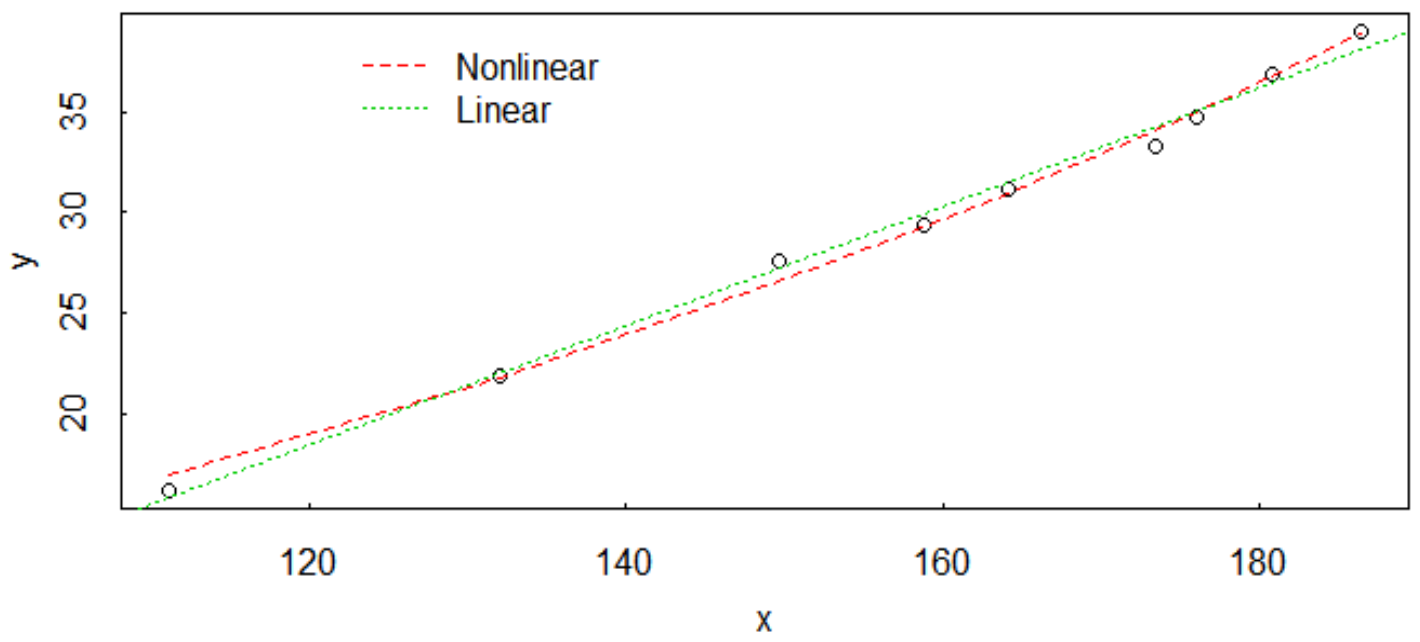

(a) Calibration data

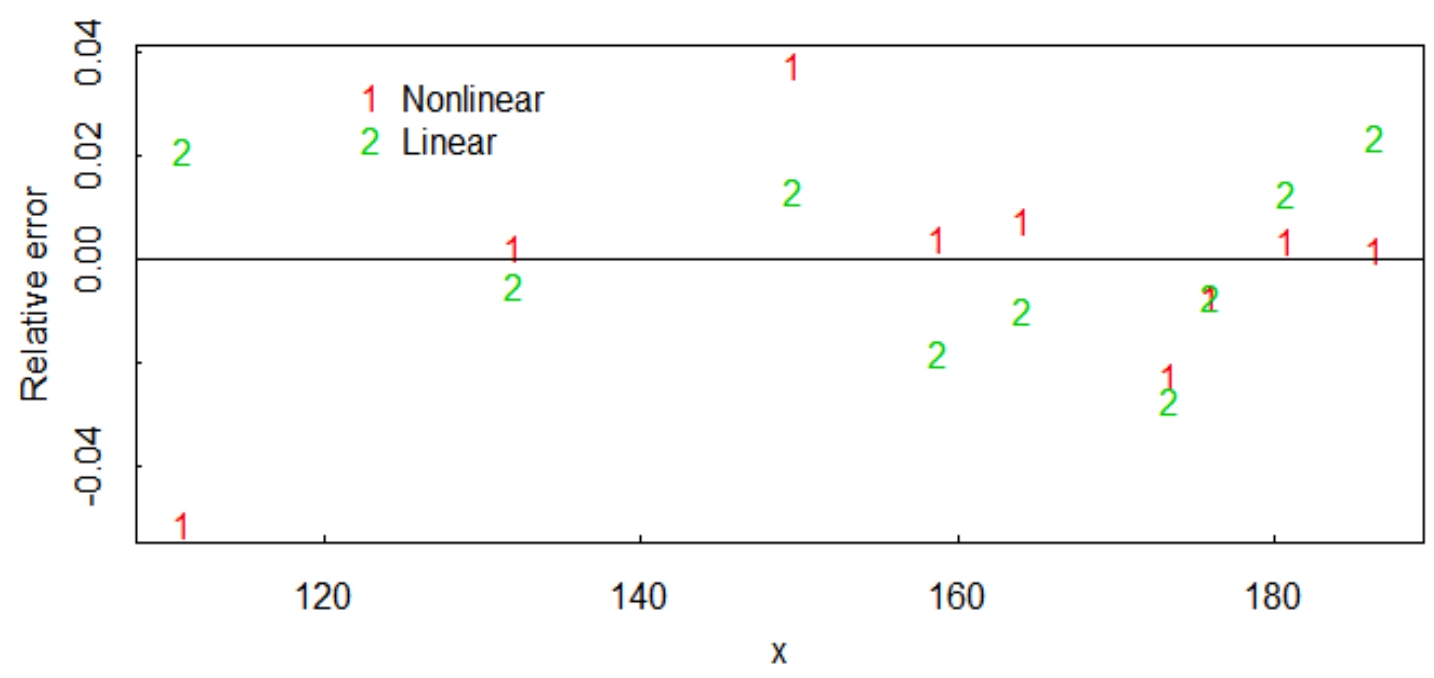

(b) Relative errors 


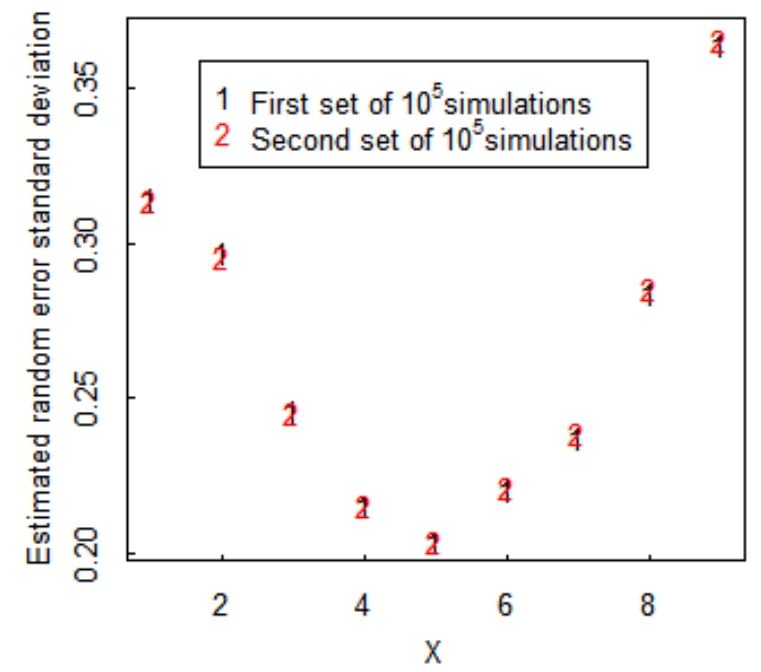

(a) Adjust for EIP

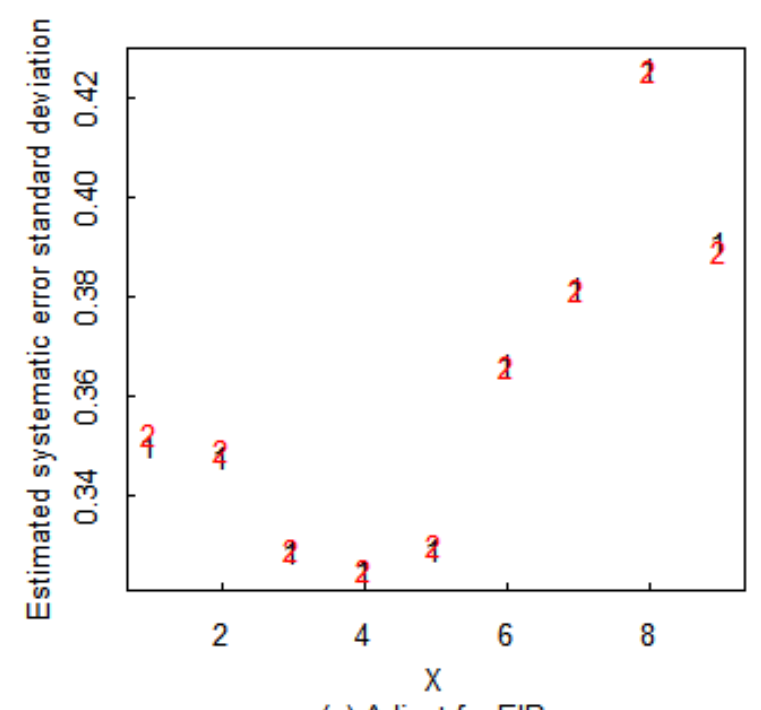

(c) Adjust for EIP

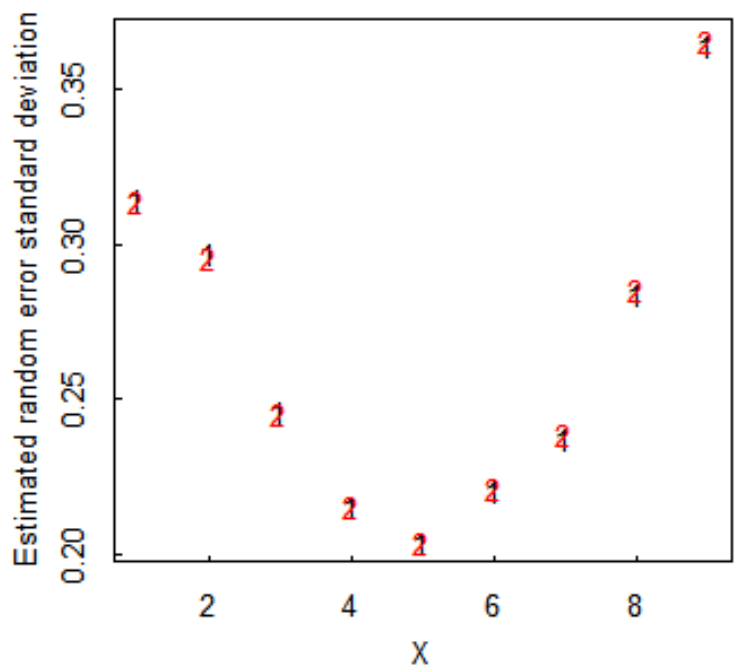

(b) Do not adjust for EIP

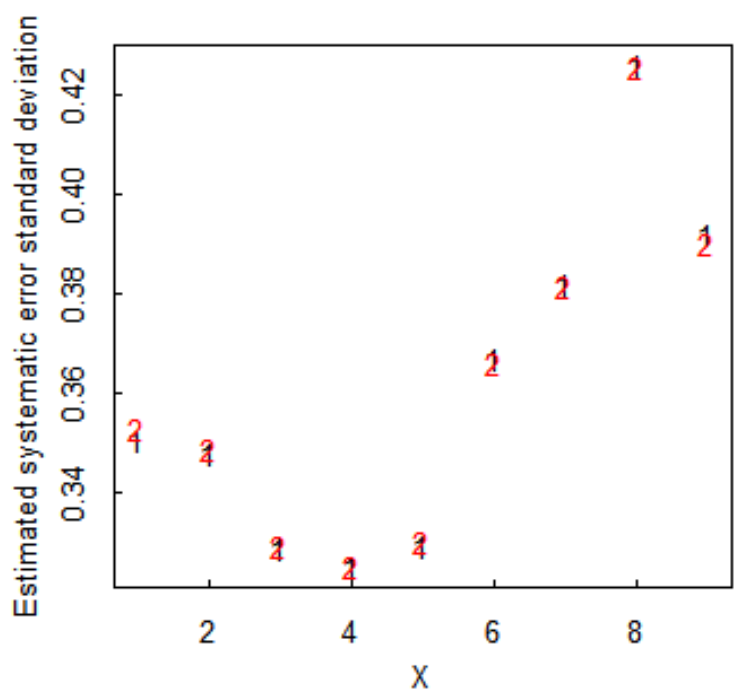

(d) Do not adjust for EIP 